\title{
USE OF THE ULBRICHT SPHERE IN MEASURING REFLECTION AND TRANSMISSION FACTORS
}

\author{
By Enoch Karrer
}

\section{ABSTRACT}

A brief historical survey is given of the methods and instruments used in measuring the reflection factor of surfaces. The theory of the infinite luminous plane is discussed at some length in connection with the Nutting absolute reflectometer, which is based on this theory. Recent suggestions of the use of the Ulbricht sphere are noted and several new ways of using it are described. A reflectometer based on one of the latter ways is described in detail. This consists in a combination of the sphere with the Martens polarization photometer. The surface whose reflection factor is sought is laid over an aperture in the sphere. The brightness of it is compared with the brightness of the sphere wall. By screening the test surface from the first reflected light this comparison gives directly the unknown reflection factor. The theory of this use of the sphere is given in full. The reflection factor of a block of magnesium carbonate is shown to be 98.7 per cent by means of this instrument.

A transmissometer based on the same theory is also described. It is also pointed out that the spectral reflection and transmission factors may be obtained in this way as a step toward standardization of methods.

A simpler and less expensive reflectometer is also described, that will enable one to obtain the reflection factor of surfaces to within ro to 15 per cent.

\section{CONTENTS}

I. Introduction

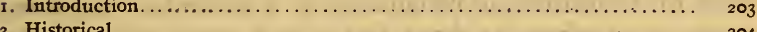

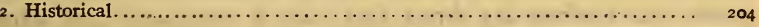

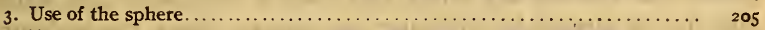

4. Theory and use of infinite planes................. 2 ro

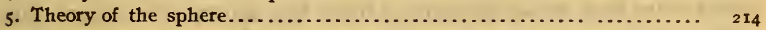

6. Description of the present reflectometer $\ldots \ldots \ldots \ldots \ldots \ldots \ldots \ldots \ldots \ldots \ldots$

7. General comments on methods of measuring reflection and transmission factors, and on standards. . . . . .

8. A simpler instrument for commercial use.................. 220

9. A transmissometer. . . . . . . . . .

10. Use of reflectometer and transmissometer with monochromatic light..... 225

\section{INTRODUCTION}

The purpose of this article is to present a general survey of the theory of the methods of measurement of the reflection factor of substances, and to describe in detail some new pieces of apparatus for the measurement of this quantity and for the measurement of the transmission factor. ${ }^{1}$

\footnotetext{
1 The reflection and the transmission factors for light are formally defined by the Illuminating Engineering Society (report of committee on nomenclature and standards, 1918). The terms are here used in accordance with these definitions, with the understanding, however, that they are determined under conditions of diffused light. Other symbols used in this article conform also to the Illuminating Engineering Society nomenclature.
} 
The apparatus described in greatest detail is a reflectometer consisting of a photometric sphere and the Martens polarization photometer. The novelty in this suggestion lies not in the use of this particular photometer in the study of the transmission and reflection by bodies nor in the use of the sphere in this connection, but in the use of the sphere in a way that is in accord with the simple theory of the sphere, and that, in conjunction with a photometer, such as the Martens, allows an absolute determination of either the transmission or reflection factor in one observation.

\section{MISTORICAL}

The measurement of reflection factors for surfaces that reflect more or less diffusely has received considerable attention. Thaler ${ }^{2}$ made a thorough test of the behavior of a few substances in this respect. Although he was not interested in the total diffuse reflection factors, his data ${ }^{3}$ for a surface smoked with magnesium oxide determine values between 0.89 and 0.90 .

Sumpner ${ }^{4}$ made use of the increased brightness of the walls resulting when a lamp was introduced within an inclosure, to study reflection and transmission by substances. Rosa and Taylor ${ }^{5}$ used the same phenomenon with the sphere to obtain the reflection factor of their sphere paint. The method depends upon the comparison of illumination at a particular point from a given source when the direct flux only is considered and when this flux is altered by inclosing the source. Since the inclosure affects the flux in all directions from the source, the direct flux, with which comparison is made, must be the average direct flux from the source.

Gardner ${ }^{6}$ gives data, obtained in The Electrical Testing Laboratories by the use of the sphere in a manner suggested by Little, on the reflection factors of colored surfaces. This method is relative, consisting of the measurement of the brightness of the sphere walls when illuminated by the test or standard surface placed successively inside the sphere. The test or standard surface only is illuminated directly by a source that is most conveniently placed outside the sphere.

\footnotetext{
'Ann. d. Phys., 316, p. 996; 1903. Good for reference to earlier work pertaining to Lambert's law. See also in this connection Mascart, "Traite d'optique," 3, p. 213.

Ann. d. Phys., 316, p. roo6, Tahle 1. (There appears to be a typographical error here in the record of angles.)

1" The diffusion of light," Phil. Mag., 35, p. 81; 1893.

s "The integrating photometric sphere, its construction and use," Trans. Illum. Eng. Soc., 2, p. 453: 1916.

o " The light-reflecting values of white and colored paints," J. Franklin Inst., 181, p. 99; $r 916$.
} 
Nutting ${ }^{7}$ has made some interesting and important considerations on an instrument for determining the reflecting power of opaque bodies. This instrument was a very portable reflectometer, depending on no standards, and had the unique property of requiring no light source as a necessary adjunct. Any general illumination at the place where measurements were made might suffice. Because this reflectometer bears some semblance to that described in this article, some parts of the underlying theory are given below. The sources of error that exist in the Nutting reflectometer will not be dwelt on here. They have been pointed out by others. ${ }^{8}$

\section{USE OF THE SPHERE}

Luckiesh ${ }^{\theta}$ has more recently called attention to the unsatisfactory state of the measurement of reflection factors, pointing out the desirability of arriving at some standard methods, and suggesting the use of the sphere in the following manner: Around a translucent sphere are distributed several light sources, and the whole is surrounded by a white-walled box. The surface, the reflection factor of which is sought, fills a small aperture in the sphere. The brightness of the test surface is measured by matching the brightness of it with that of the field in an auxiliary photometer. The proposed method is good, but it involves the use of all the photometric accessories required in the ordinary use of the sphere, and requires, further, a standard reflecting surface. The means employed of illuminating the sphere walls would have to be altered somewhat in order that full advantage may be taken of the theory of the sphere. In any case, however, the use of the principle of the sphere has decided advantages over the laborious method of measuring the reflection factor for various directions, and then by integration, graphically or otherwise, deriving the total reflection factor.

Much credit is due Taylor ${ }^{10}$ for pointing out several ways by which the sphere may be used to determine the absolute reflection factor. The most obvious ways (including those of Mr. Taylor and others) of using the sphere to measure the reflection factor may be enumerated as follows:

I. By comparison of the illumination due to reflected flux alone with that due to both direct and reflected flux. If $E_{1}$

\footnotetext{
7 Trans. Illum. Eng. Soc., 7, p. 412; 1912.

8 "Measurement of diffuse reflection factors," J. Am. Op. Soc., 4, p. 9, 1920; B. S. Sci. Papers, Nos, 391 and 405 ; and Gen. Elec. Rev., 23, p. 72, 1920.

" "Measurement of the reflection factor," Electrical World, 69, p. 958; 1927.

10 "Measurement of diffuse reflection factors," J. Am. Op. Soc., 4, p. 9; 1920. Also B. S. Sci. Papers, Nos. 391 and 405 .
} 
is the illumination due to the average direct flux and $E_{2}$ that due to the reflected flux, then

$$
\frac{E_{2}}{E_{1}+E_{2}}=\rho
$$

This is the method used by Sumpner and Rosa and Taylor, as described above, to obtain the absorption factor $(\alpha)$ directly and derive therefrom the reflection factor. In this case

$$
\frac{E_{1}}{E_{1}+E_{2}}=a=1-\rho
$$

Sumpner, however, used an inclosure in the form of a hollow cylinder rather than in the form of a hollow sphere. Sumpner also used this method to obtain the transmission factor of substances. It is applicable only to materials that can be made into the form of a sphere, or that may be applied to the inner sphere walls.

2. By illuminating sphere walls as a whole indirectly by first illuminating a small portion of them. Brightness of the walls is measured when complete and when a known portion of the sphere is removed. This is the basis of a proposed reflectometer by Taylor ${ }^{11}$ to obtain the reflection factor of painted sphere walls. The reflection factor $\rho$ of the sphere wall is given by the equation

$$
\begin{gathered}
\rho^{2}\left[q(p+q-\mathrm{I})+\frac{b}{b_{0}} p(\mathrm{I}-q)\right]+\rho(\mathrm{I}-q)\left[q+(p+q-\mathrm{I})\left(\mathrm{I}-\frac{b}{b_{0}}\right)\right] \\
+(\mathrm{I}-q)^{2}\left(\mathrm{I}-\frac{b}{b_{0}}\right)=0
\end{gathered}
$$

where $q$ is the percentage of the sphere surface cut off by the hole; $p$, the percentage of area added to the sphere wall when the hole is covered by a plane surface; $b$ and $b_{0}$ are brightnesses of the sphere wall due to reflected light when the test surface has a reflection factor of $\rho$ and o, respectively. A particular application of this method was made by Benford ${ }^{12}$ for the determination of the reflection factor of magnesium carbonate as follows: The brightness $\left(b_{1}\right.$ and $\left.b_{2}\right)$ of the sphere wall was measured after the removal in succession of two known fractional parts $\left(1-p_{1}\right)$ and $\left(1-p_{2}\right)$ of the sphere. The reflection factor is given by

$$
\rho=\frac{b_{1}-b_{2}}{b_{1} p_{1}-b_{2} p_{2}}
$$


This method, as the previous one, is applicable only when the test substance can be made into a sphere or can be applied to the inner sphere walls. It has one very desirable feature, in that not only is the illumination diffuse but the reflected flux which is measured is also, and the whole of it is directly contributory to the results. The degree to which the flux is diffused depends upon the test substance itself, for the latter constitutes the whole of the sphere wall. It must be applied with caution when the test substances are any other than highly diffusing.

3. By method (2), modified so that the specimen need not be the whole of the sphere wall but merely a small known portion of it. In this case the reflection factor $\rho_{x}$ of the test surface is given by

$$
\rho_{\mathrm{x}}=\frac{\mathrm{I}-\frac{b_{0}}{b_{\mathrm{x}}}}{\frac{q}{\mathrm{I}-q} \cdot \frac{b_{0}}{b_{\mathrm{x}}}+\frac{\rho p}{\rho p+(\mathrm{I}-q)(\mathrm{I}-\rho)}}
$$

where $p, q, \rho$, and $b_{0}$ have the same meaning as in the method (2), and $b_{x}$ is the brightness of sphere wall when the test surface is in place. The equation given under method (2) is derived from the present one when $\rho_{x}=\rho$. This method retains the desirable diffuseness in both incident and reflected light and obviates the restrictions of method (2) above, but the sensitivity of it depends upon the value of the reflection factor measured.

4. By use of part sphere, test (or standard) surface constituting a known portion of the sphere wall is illuminated directly. The standard is a specimen having the reflection factor of the sphere wall. The brightness of the sphere wall is measured first when illuminated by the specimen, then when illuminated by the standard. The reflection factor $\left(\rho_{\mathrm{z}}\right)$ of the test surface is given by ${ }^{13}$

$$
\rho_{\mathrm{x}}=\frac{\frac{b_{\mathrm{x}}}{b}}{\mathrm{I}-\frac{\rho^{2} p\left(\mathrm{I}-\frac{b_{\mathrm{x}}}{b}\right)}{\mathrm{I}-q+\rho(p+q-\mathrm{I})}}
$$

$b_{x}$ and $b$ are the values of the brightness of the sphere wall when the aperture is covered by the test surface and by the standard surface respectively. This method was one adopted by Taylor

18 J. Am. Op. Soc., 4, D. 16; 1920. 
in preference to the preceding one because it is more accurate and more sensitive.

5. By use of the complete sphere, the specimen being placed inside and illuminated directly (preferably by a narrow beam of light projected through a window). The standard is a specimen of known reflection factor. Two measurements of brightness of the sphere wall are necessary, first, when illuminated by the specimen, second, when illuminated by the standard. This method was suggested and used by Little, ${ }^{14}$ whose standard was a magnesium-carbonate surface. The ratio of these gives the reflection factor of the sample in terms of the standard. This method may be improved by eliminating the standard surface. This may be accomplished by screening the test surface from the observation window, then comparing the brightness of the sphere wall, first, when illuminated by the sample, and second, when illuminated by illuminating a small portion of the wall. In the latter case no screen is interposed.

6. By use of the complete sphere, the specimen being placed inside and illuminated by the sphere wall. The brightness of the sample and of the sphere wall are compared by one observation. The sphere wall may be illuminated by illuminating a small portion of it. The reflection factors are in terms of that of the sphere wall. If the test surface only is screened from the illuminated portion the method is an absolute one.

7. By directly illuminating the specimen (constituting a negligible portion of the sphere wall). The walls are illuminated by the specimen. The brightness of the sphere wall at a point screened from the test surface is measured, first, as resulting from the illumination of the test surface, and second, as resulting from an equal illumination of a portion of the sphere walls unscreened from the observed spot. The ratio of these is the reflection factor of the surface. This is the method embodied in the reflectometer more recently designed by Taylor. ${ }^{15}$ No standard is required.

8. By illuminating the specimen (constituting a negligible portion of the sphere) by means of the sphere walls, which in turn are illuminated by a narrow beam admitted through a small window. The specimen is screened from the illuminated spot.

\footnotetext{
14 "The light-reflecting values of white and colored paints," J. Franklin Inst., 181, p 99; 1916.

16 "A simple portable refiectometer," A. H. Taylor, Trans. Illum. Eng. Soc., paper read at 14 th annual convention, Illum. Eng. Soc,; 1920. Also B. S. Sci. Papers, No. 405.
} 
No standard is required. This method was used by Sharp and Little. ${ }^{16}$ These authors showed that the reflection factor of a mirror as well as that of other surfaces may be correctly measured by this method. It may also be pointed out that all movements of the photometer in making the measurements requirednamely, of the brightness of, first, the specimen and, second, the sphere wall-may be avoided by employing a mirror of known reflection factor. In some experimental work with this method the author used a glass prism, by the rotation of which either the specimen or the comparison spot could be brought into view.

9. By method (8) just described, simplified by the use of a Martens polarization photometer. One measurement, comparing the brightness of the specimen with that of the sphere wall, will then suffice. This is the method to which special attention is called in this article. It is the method embodied in the reflectometer described in detail below. It is suggestive of the best combination (for certain photometric work) in which the sphere is used in a manner that takes advantage of its properties and at the same time affords simplicity of parts, compactness, and directness.

Io. Still other methods may be designed. For example, by using the specimen as a known portion of sphere wall. Another known portion removed. Two measurements of brightness of sphere wall are required, first, when sphere is complete, and, second, when the known portion is removed. This has the advantage that both the incident and the reflected light are diffused. The degree to which the light is diffused depends in this case and in method (3) above, not upon the specimen as in methods (I) and (2) above, but upon the sphere wall.

II. By modification of the foregoing methods, using two spheres simultaneously, so that only one measurement is neces sary.

I2. By use of a new method of obtaining the reflection factor of a substance that may be applied to the walls of the sphere or that may be made into a sphere, as suggested by the equation given below under the theory of the sphere. The relation between the brightness, $b_{s}$, of the sphere wall resulting when a portion, $p$,

\footnotetext{
${ }^{16} \mathrm{C}$. H. Sharp and W. F. Little, "Measurement of reflection factors," Trans. Illum. Eng. Soc., papet read at ith annual convention, Illum. Eng. Soc.; 1920. See also paper by A. H. Taylor, presented at the same time, and discussion by Karrer.
}

$54147^{\circ}-21-2$ 
of it is made luminous and the brightness, $b$, of the luminous portion is given by

$$
b_{\mathrm{s}}=p b \cdot \frac{\rho}{\mathrm{I}-\rho} .
$$

The reflection factor $\rho=\frac{r}{p+r}$, where $r=\frac{b_{\mathrm{s}}}{b}$. The quantities $p, b$, and $b_{\mathrm{s}}$ may be measured. In particular, if $p$ were changed until $b_{8}=b$, then $\rho=\frac{1}{I+p}$. It seems that this method is simpler than method (2), where portions of the sphere are removed, and simpler than method $(I)$, where it is required to know the mean spherical candles of the source.

One simple way suggests itself whereby no beam of light need be projected into the sphere, namely, a plate of diffusing glass is illuminated on one side so that the brightness of the reverse side is $b$. The plate with the latter side toward the sphere is then placed against the sphere aperture of known dimensions. Finally the brightness of the sphere walls produced by this luminous plate is measured. This arrangement would readily allow of a variable aperture into the sphere.

Furthermore, the method of illuminating either the specimen or the sphere wall in some instances may be different from that stated above; for example, in method (8) above, the sphere wall might be illuminated by transmitted light as indicated by Luckiesh. ${ }^{17}$

\section{THEORY AND USE OF INFINITE PLANES}

The Nutting instrument is based upon the principle of the infinite luminous plane. The illumination ${ }^{18}$ on a plane exposed to the flux from an infinite parallel plane source of uniform brightness $b$ (lamberts) is $\pi b$, provided every element of the infinite plane radiates according to the cosine law. If a small reflecting surface be exposed to this illumination, it will reflect per unit area at the rate of $\pi \rho b$ lumens, where $\rho$ is the reflection factor of the surface. Its brightness, therefore, is $\rho b$. Hence the ratio of the brightness of the small plane to that of the infinite plane is the reflection factor sought. The surface under test should be small enough not to change the brightness of the luminous plane. Such simple conditions are not realized, however, in

17 "Measurement of diffuse reflection factors," Jour. Am. Op. Soc., 4, p. 9, 1920; B. S. Sci. Papers, Nos. $39 \mathrm{r}$ and 405; and Gen. Elec. Rev., 23, D. 72, 1920.

18 B. S. Bull., 6, p. 553; x910 
practice. In case of the Nutting reflectometer, two planes of the same size constitute the radiating and the receiving planes. In such a case, if we assume them infinite, their resulting brightness is readily calculable.

The brightness of the planes is increased by the multiple reflection of light between them. Let $S$ (Fig. I) be the infinite plane of brightness $b$, illuminating the infinite plane $T$, and let the reflection factors of these planes be $\rho_{2}$ and $\rho_{1}$, respectively. As a result of the direct illumination from $S, T$ will be of uniform brightness $\rho_{1} b$, and will reradiate onto $S$ at the rate $\pi \rho_{1} b$. As a result of

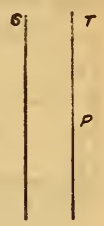

Fig. I this $S$ will have its brightness increased by an amount of $\rho_{1} \rho_{2} b$. The plane $S$ will in turn increase its illumination on $T$ by an amount, $\pi \rho_{1} \rho_{2} b$. The total illumination $E_{\mathrm{T}}$ of surface $T$ will be

$$
E_{\mathrm{T}}=\pi b+\pi \rho_{1} \rho_{2} b+\pi \rho_{1}{ }^{2} \rho_{2}{ }^{2} b+\cdots \cdot \text { etc. }
$$

The brightness of $T$ is, therefore,

$$
b_{\mathrm{T}}=\rho_{1} b\left(\mathrm{I}+\rho_{1} \rho_{2}+\rho_{1}{ }^{2} \rho_{2}{ }^{2}+\cdots \cdots+\rho_{1}{ }^{\mathrm{n}-1} \rho_{2}{ }^{\mathrm{n}-1}+\cdots\right)=\frac{\rho_{1} b}{\mathrm{I}-\rho_{1} \rho_{2}} .
$$

For the surface $S$ we obtain the brightness

$$
\begin{aligned}
& b_{\mathrm{B}}=b\left(\mathrm{I}+\rho_{1} \rho_{2}+\rho_{1}{ }^{2} \rho_{2}{ }^{2}+\cdots \cdots+\rho_{1}{ }^{\mathrm{n}-1} \rho_{2}{ }^{\mathrm{n}-1}+\cdots\right)=\frac{b}{\mathrm{I}-\rho_{1} \rho_{2}} . \\
& b_{\mathrm{T}}=\rho_{1} .
\end{aligned}
$$

In order to approach the condition of infinite planes, and at the same time retain limits consistent with a portable instrument, Nutting bounded the planes with a mirror.

The effect of the mirror is virtually to extend by multiple reflection the planes indefinitely. If the mirror were nonabsorbing, the finite planes bounded by it would give effects similar to infinite planes. The theory of them then would be also as simple as that of infinite planes. The mirror, however, will reflect only a certain proportion $\rho_{\mathrm{m}}$ of the flux falling upon it, and as a consequence, instead of an infinite plane with uniform brightness, we have an infinite plane composed of concentric zones of varying brightness. It becomes more difficult to calculate the illumination due to such a luminous plane at any point in space. This will be seen by considering, for sake of simplicity, the illumination at a point $P$ which lies on the line perpendicular to the plane at the common 
center of all the zones. Let $S$, Fig. 2, be a luminous disk of diameter $2 a$ and of uniform brightness $b$, surrounded by an annular area of uniform brightness $\rho_{\mathrm{m}} b$. For the present the annular area

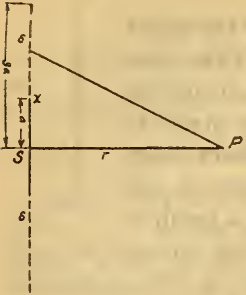

Fig. 2 may be regarded as the reflection of the disk in a surrounding circular mirror which reflects an amount $\rho_{\mathrm{m}}$. The width of the annular area is therefore $2 a$; its brightness $\rho_{m} b$. The illumination due to the disk $S$ at the point $P$ is

$$
E_{\mathrm{d}}=\pi b \int_{0}^{\mathrm{a}} \frac{2 r^{2} x d x}{\left(r^{2}+x^{2}\right)^{2}}=\frac{\pi b a^{2}}{r^{2}+a^{2}}
$$

where $r$ is the distance of the point $P$ from the disk. ${ }^{19}$

Similarly the illumination at $P$ due to the annular portion ss of the disk of brightness $b_{1}$ is

$$
E_{1}=\pi b_{1} \int_{a}^{c_{3}} \frac{2 r^{2} x d x}{\left(r^{2}+x^{2}\right)^{2}}=\frac{8 \pi b_{1} a^{2} r^{2}}{\left(r^{2}+9 a^{2}\right)\left(r^{2}+a^{2}\right)} .
$$

So that the total illumination at $P$ is the sum of these

$$
E_{\mathrm{t}}=\frac{\pi b a^{2}}{\left(r^{2}+a^{2}\right)}\left(\mathrm{x}+\frac{8 \rho_{\mathrm{m}} r^{2}}{r^{2}+9 a^{2}}\right)
$$

where $\rho_{\mathrm{m}}=\frac{b_{1}}{b}$.

If the disks were of uniform brightness, that is, $\rho_{m}=1.00$,

$$
E_{t}=2 \pi b r^{2} \int_{0}^{3 a} \frac{x d x}{\left(r^{2}+x^{2}\right)^{2}}=\frac{9 \pi b a^{2}}{\left(r^{2}+9 a^{2}\right)} .
$$

In the actual case there is an infinite number of concentric zones which contribute to the illumination at $P$ and the resultant effect at $P$ may be represented by a summation of such integrals as that given above for the annular zone ss due to the first order of reflection. The effect of the $n$th order of reflection may be written after noting that the position and boundary of the $n$th image are determined by its radii, which are $a(2 n-\mathrm{I})$ and $a(2 n+\mathrm{I})$. 
The illumination at $P$ due to the $n$th reflection is then

$$
E_{\mathrm{n}}=\pi b \rho_{\mathrm{m}}^{\mathrm{n}} \int_{a(2 n-\mathrm{r})}^{\frac{2(2 n+1)}{\left(r^{2}+x^{2}\right)^{2}}}=\frac{8 \pi b a^{2} r^{2} n \rho_{\mathrm{m}}{ }^{\mathrm{n}}}{\left[r^{2}+(2 n-\mathrm{I})^{2} a^{2}\right]\left[r^{2}+(2 n+\mathrm{I})^{2} a^{2}\right]} .
$$

The illumination due to all zones is

$$
E_{\mathrm{a}}=\sum_{n=1}^{n=\infty} \pi b \rho_{\mathrm{m}} \int_{a(2 n-\mathrm{x})}^{\mathrm{a}^{\mathrm{a}}(2 n+\mathrm{r})} \frac{2 r^{2} x d x}{\left(r^{2}+x^{2}\right)^{2}}=\sum_{n=\mathrm{x}}^{n=\infty} \frac{8 \pi b a^{2} r^{2} n \rho_{\mathrm{m}}{ }^{\mathrm{n}}}{\left[r^{2}+(2 n-\mathrm{I})^{2} a^{2}\right]\left[r^{2}+(2 n+\mathrm{I})^{2} a^{2}\right]} .
$$

And the total illumination becomes

$$
E_{\mathrm{t}}=E_{\mathrm{d}}+E_{\mathrm{a}}=\pi b a^{2}\left\{\frac{\mathrm{I}}{r^{2}+a^{2}}+8 r^{2} \sum_{n=1}^{n=\infty}\left[\frac{n \rho_{\mathrm{m}}^{\mathrm{n}}}{\left.\left[r^{2}+(2 n-\mathrm{r})^{2} a^{2}\right]\left[r^{2}+(2 n+\mathrm{r})^{2} a^{2}\right]\right\}}\right.\right.
$$

A few terms of this expression may be written out for the sake of the discussion.

$$
\begin{gathered}
E_{\mathrm{t}}=\pi b a^{2}\left[\frac{\mathrm{I}}{a^{2}+r^{2}}+8 r^{2}\left\{\frac{\rho_{\mathrm{m}}}{\left(r^{2}+9 a^{2}\right)\left(r^{2}+a^{2}\right)}+\frac{2 \rho_{\mathrm{m}}^{2}}{\left(r^{2}+25 a^{2}\right)\left(r^{2}+9 a^{2}\right)}+\right\}\right. \\
+8 r^{2}\left\{\frac{n \rho_{\mathrm{m}}}{\left\{r^{2}+(2 n-\mathrm{I})^{2} a^{2}\right]\left[r^{2}+(2 n+\mathrm{I})^{2} a^{2}\right]}\right. \\
\left.\left.+\frac{(n+\mathrm{I}) \rho_{\mathrm{m}}{ }^{\mathrm{n}+1}}{\left[r^{2}+(2 n+3)^{2} a^{2}\right]\left[r^{2}+(2 n+\mathrm{I})^{2} a^{2}\right]}+\cdots \text { etc. }\right\}\right]
\end{gathered}
$$

This series is, in general, very rapidly converging. The ratio of the $(n+r)$ th to the $n$th term being

$\rho_{m} \frac{n+\mathrm{I}}{n}\left\{\mathrm{I}-\frac{(8+16 n) a^{2}}{\gamma^{2}+(2 n+3)^{2} a^{2}}\right\}$, which approaches the value $\rho_{\mathrm{m}}$ for large values of $n$, but is always less than $\rho_{\mathrm{m}}$ if $a$ is large compared with $r$ (say, $a=3 r$ or more). The value of this ratio for the first and second terms is $\rho_{\mathrm{m}}$ if $a=2 r$. The effect of the illumination due to the $n$th reflection compared with that of the disk alone is:

$$
e=\frac{8 r^{2} n \rho_{\mathrm{m}}{ }^{\mathrm{n}}\left(a^{2}+r^{2}\right)}{\left[r^{2}+(2 n+\mathrm{I})^{2} a^{2}\right]\left[r^{2}+(2 n-\mathrm{r})^{2} a^{2}\right]} .
$$

If $a / r$ is assumed to be 5 , and $\rho_{\mathrm{m}}$ to be Ioo, then the effect of the first and second images is about 5 per cent of that of the disk, and the effect of the first ro images is slightly less than 6 per cent of that of the disk. It is evident that as the reflection factor of the mirror approaches unity the disk as extended by its images becomes more like an infinite plane. 
From the equation for $E_{\mathrm{t}}$ it is also seen that as $r$ becomes very small the illumination at $P$ approaches that given by an infinite plane of uniform brightness, $E_{\mathrm{t}}=\pi b$.

The foregoing considerations show that the theoretically simple conditions are not at all attained in the use of infinite planes as in the Nutting instrument. An improvement could be effected by making the test surface only a small portion of one of the planes. This would also be the case if one were to consider the instrument from the point of view of a finite inclosure rather than from the viewpoint of infinite planes.

A very good approach to two infinite planes can be had by two concentric spherical surfaces. Either of the surfaces may be made luminous with uniformity of brightness. The test surfaces may be made a part or the whole of the nonluminous sphere. A sphere uniformly bright illuminates all points on any given concentric spherical surface equally, and more nearly like an infinite plane as the points approach the surface of the luminous sphere. $^{20}$ Of course, the last condition would be impossible to realize in a practical instrument. Yet this interposes no hindrance to the practical use of two concentric spherical surfaces as described. For when the surfaces are separated any finite distance the relative distribution of the illumination from the one on to the other remains unaltered. The magnitude only of the illumination changes, becoming less than that which is given by an infinite plane in the ratio of the square of the radius of the sphere to the square of the distance from the center of the sphere to the concentric spherical surface. (The latter statement applies when the inner sphere is the luminous one.) Just what other difficulties would arise in the actual execution of this arrangement can not be foretold.

It is of interest to note that the flux between two infinite parallel planes depends upon the reflection factors of them in a manner quite different from that in which the flux in a sphere depends upon the reflection factor of the sphere wall.

\section{THEORY OF THE SPHERE}

For an inclosure we find the most satisfactory case to consider is that of the hollow sphere, for although the flux in any inclosure is everywhere the same ${ }^{21}$ if the walls of the inclosure are uniformly bright, it is only in case of the sphere where this latter condition of uniformity of brightness is easily obtainable. 
In the sphere, whose walls reflect according to Lambert's law, every element of surface illuminates every other element to the same degree ${ }^{22}$ no matter how the wall of the sphere or any portion of it is directly illuminated. This property of the spherical inclosure was first discovered by Sumpner. ${ }^{23}$ It was rediscovered by Ulbricht, ${ }^{24}$ who first demonstrated its great practical importance to photometry. So that, if the surface of the sphere has a uniform reflection factor and if a small portion of it is made luminous, the whole sphere will be uniformly bright, except for this spot which may be of negligible proportions. The brightness may be calculated as follows:

Let the illuminated spot of area $A$ have an average brightness of $b$. It radiates $\pi b A$ lumens. Owing to multiple reflections this flux will accumulate to a value $F$ such that the rate of loss $\alpha F$ equals the rate of input $\pi b A$, where $\alpha$ is the absorption factor.

$$
\begin{aligned}
\alpha F & =\pi b A \\
F & =\frac{\pi b A}{\alpha}=\frac{\pi b A}{\mathrm{I}-\rho} .
\end{aligned}
$$

This was first derived by Mascart. ${ }^{25}$ This reasoning may be employed for any inclosure whose walls obey Lambert's cosine law of emission. When the walls are not uniformly bright, $b$ refers to the average brightness.

The illumination on every other element of the surface of the sphere will be

$$
E=\frac{b A}{4 r^{2}} \cdot \frac{I}{I-\rho} .
$$

Hence the brightness of every element of the surface of the sphere (except $A$ ) is

$$
\frac{\rho b A}{4 \pi r^{2}} \cdot \frac{\mathrm{r}}{\mathrm{I}-\rho} \equiv b_{\mathrm{s}}
$$

The ratio of the area of the luminous spot to the area of the sphere is

So that

$$
\frac{A}{4 \pi r^{2}} \equiv p
$$

$$
b_{\mathrm{s}}=\frac{p b \rho}{\mathrm{I}-\rho}
$$

92 Liebenthal, Praktische Photometrie, p. $30 \mathrm{x}$

23 "The diffusion of light," Phil, Mag., 35, p. 81; 189.3 .

34 Electrotechn. Zs. 21, p. 595,$1900 ; 26$, p. 512,1905

15 Palaz, Traité de Photométrie Industrielle, translation by the Pattersons, p. 297. 
If now for a small portion of the sphere surface there is substituted another nonluminous surface whose reflection factor is $\rho_{\mathbf{x}}$, the brightness $b_{x}$ of the latter will be

and

$$
b_{\mathrm{x}}=\frac{\rho_{\mathrm{x}} b p}{\mathrm{I}-\rho}=\frac{\rho_{\mathrm{x}} b_{\mathrm{B}}}{\rho}
$$

$$
\frac{b_{\mathrm{x}}}{b}=\frac{\rho_{\mathrm{x}}}{\rho}
$$

The reflection factor of any specimen is given in terms of that of the sphere wall. To make it possible to obtain the reflection factor of the specimen absolutely, certain conditions must be imposed upon the manner in which the specimen is illuminated. If all of the flux illuminating the specimen is reduced by a factor $\rho$, the brightness of the specimen will also be reduced in the same proportion.

Then $b_{\mathrm{x}}$ becomes $b_{x}^{\prime}=\rho b_{x}=\rho_{x} b_{8}$.

and $\frac{b_{x}^{\prime}}{b_{\mathrm{S}}}=\rho_{\mathrm{x}}$.

This result is achieved by interposing a screen between the illuminated spot and the test surface. The screen is just sufficiently large to enable it to intercept all of the flux reflected from the former in the direction of the latter. The illuminated spot must be small in order that no appreciable illumination due to the second and higher order of reflections would come to the test surface if the screen were not present. The new surface also is assumed to be so small that its presence does not alter the brightness of the sphere walls.

All of these theoretical conditions can be attained to a satisfactory degree in practice. This is the idea underlying several uses of the sphere in reflectometry listed above and in particular the reflectometer ${ }^{26}$ depicted in Fig. 3 .

\section{DESCRIPTION OF THE PRESENT REFLECTOMETER}

The reflectometer sketched in Fig. 3 is essentially a sphere, having, firstly, an aperture which may be closed by any surface whose reflecting factor is sought, secondly, a means for comparing directly the brightness of the test surface with that of the

26 This reflectometer was shown belore the Illuminating Engineering Society at Cleveland, Oct. 5, 1920. A general description of it will appear in Trans. Illum. Eng. Soc, as discussion of the papers: "Measurement of reflection factor," C. H. Sharp and W. F. Little; and "A simple portable reflectometer of the absolute type," A. H. Taylor 
sphere surface, such as a Martens ${ }^{27}$ photometer, and thirdly, a lamp for illuminating a small portion of the sphere.

The most satisfactory photometer for comparing the brightness of two surfaces not far removed from each other is the Martens

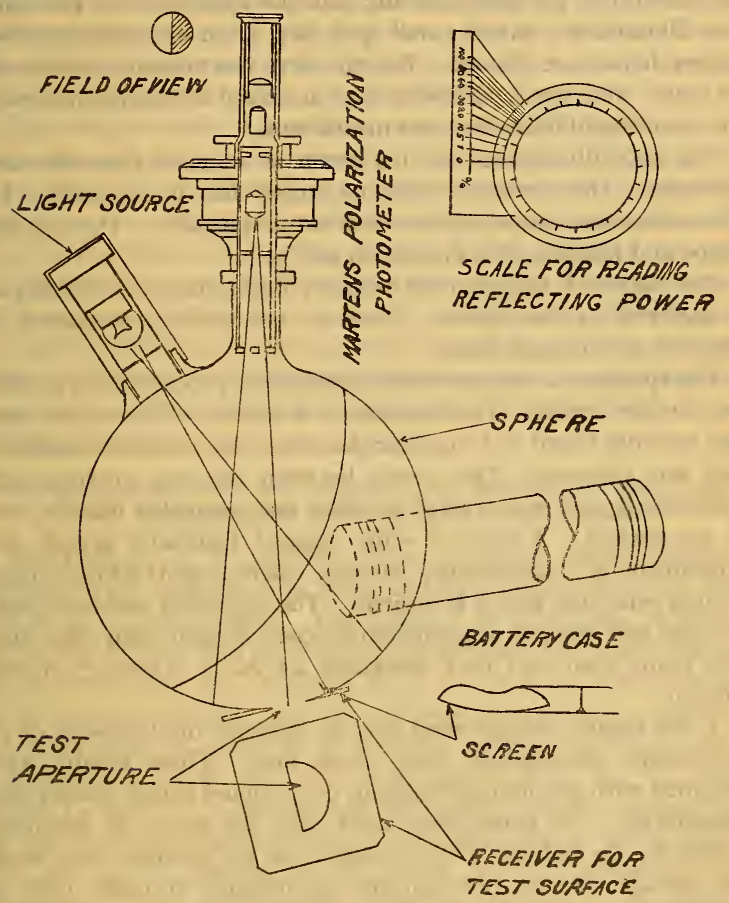

FIG. 3.-Improved Nutting reflectometer

Scale $1 / 2$ natural size

polarization photometer. It is very compact, accurate, and has an extremely good photometric field. ${ }^{28}$ Its optical parts are, in order of position in the optical path, Fig. 3, two apertures, lens, Wollaston prism, biprism, Nicol prism, and eyepiece containing

97 Phys. Zs., 1, pp. 299-303; 1900

${ }^{28}$ In spite of the fact that this instrument is not patented it is unfortunately not readily procurable. 
lens and aperture. A scale reading directly the absolute reflection factor is attached to the circular scale already a part of the Martens photometer. The lamp is a small Mazda flashlight lamp operated at 3.8 volts by a small 3 -cell flashlight battery. It is not difficult to get sufficient flux into the sphere and at the same time illuminate a rather small spot, say, $3 \mathrm{~cm}$ in diameter, when proper lenses are chosen. For the first instrument constructed no lenses were used. For ease and accuracy of setting, however, the resulting illumination was insufficient.

The spot illuminated by the beam is screened from the test aperture. This screen is made no larger than is necessary. Its dimensions and shape were determined graphically. The size and shape and location of it are shown in Fig. 3.

The battery is placed in an ordinary flash-lamp tube whose end is soldered to the sphere. Thus a very convenient handle is afforded at the same time.

The sphere is a copper-covered steel float, procurable at plumbers' supply houses. Its diameter is 6 inches. The copper coat was actually found to be undesirable when the sphere is enameled, and was removed. The screen between the test aperture and illuminated spot was riveted in place and enameled like the rest of the surface. A coat of white enamel facilitates greatly the attainment of a satisfactory surface, where a matt-white surface of high reflection factor is desired. The enameled surface is easy to paint and requires a much thinner coat of paint than otherwise. The paint used was that described by A. H. Taylor ${ }^{20}$ of this Bureau.

A few results are recorded here to indicate the behavior of an instrument constructed along these lines. These results were obtained with the first instrument, constructed rather hastily and imperfectly. No lenses were used with the lamp; no adequate holder for the battery was attached; and no provision was made for readily turning the Martens photometer through $180^{\circ}$ to eliminate any asymmetric errors. Settings were made in each of the four quadrants.

Magnesium carbonate block No. I (A. H. T.) -0.980 ;

Magnesium carbonate block No. 2 (A. H. T.) -0.972 ;

Magnesium carbonate block No. I (E. K.) -0.980 .

The first two blocks of magnesium carbonate were loaned by Mr. Taylor, who has determined the reflection factor of them by

${ }^{20}$ B. S. Sci. Papers, in preparation, "The integrating sphere," Rosa and Taylor 
various methods, arriving at 0.99 for No. I and 0.982 for No. 2. There seems to be a consistent difference. Other tests were made on a series of surfaces which had been studied by Mr. Taylor using the point by point method. Differences in the factor were usually obtained (although not always), which indicated that the above reflectometer was reading slightly low. The value obtained here for the magnesium carbonate agrees with those found first by Mr. Taylor ${ }^{30}$ and later by Mr. Benford ${ }^{31}$ and others. ${ }^{32}$

The sample needs to be neither opaque nor diffusing. It may be a clear piece of glass or mirror. This condition follows on the assumption that the hole in the sphere over which the specimen is placed is so small that its area is negligible in comparison with that of the whole sphere. In the one described above the area of the hole is about $3 \mathrm{~cm}^{2}$, or less than one-half per cent of that of the sphere. In the case of a mirror, however, precautions must be taken not to have the second reflecting surface too far removed from the opening. The angle subtended by the image of the test aperture must always be sufficiently large to fill the field of view of the photometer.

\section{GENERAL COMMENTS ON METHODS OF MEASURING REFLECTION AND TRANSMISSION FACTORS, AND ON STANDARDS}

Many aspects of the problem of measuring the diffuse reflection factor call for consideration, some of which may or may not turn out to be of any moment. For instance, in case of the reflection factor the surface may be diffusely illuminated, but in general can be observed from one direction only. Or it may be illuminated from one direction only, and the diffusely reflected flux measured. In either case the question of direction enters, concerning which nothing much can be said until more work is done; the use of the sphere is, however, a step toward uniformity and standardization. In case of the transmission factor the illumination on the specimen may be entirely diffuse and the whole of the transmitted flux measured. This can be accomplished by the use of two spheres, comparing the brightness of the walls of them, so that the question of direction may be easily eliminated in case of the transmission factor.

\footnotetext{
so A. H. Taylor, "Measurement of diffuse reflection factors and a new absolute reflectometer," J. Am. Op. Soc, 4, p. 9; 1920.

"F. A. Benford, "An absolute method for determining coefficients of diffuse reflection," Gen. Elec. Rev., 23, p. 72: 1920.

n C. H. Sharp and W. F. Little, "Measurement of reflection factors," Trans. Mlium. Eng. Soc., paper read at inth anuual convention, Illum. Eng. Soc., 1920. (See also paper by A. H. Taylor, presented at the same time, and discussion by Karrer.)
} 
Four of the methods enumerated above for determining the reflection factor-namely, (2), (3), (9), and (12)-afford diffusion in both the incident and the measured flux. The first and last of these place very serious limitations upon the nature and the form of the surface to be tested. The other two are insensitive for certain values of the reflection factor, their sensitivity depending upon this factor in a rather complicated manner. Method (I) virtually allows diffuseness in both incident flux and measured flux, but the extent to which this condition actually prevails depends upon the relative importance of the secondary flux, $E_{2}$, and therefore upon the reflection factor itself.

At all events, so far as a working standard is concerned, the procuring of a magnesium carbonate block at any market is to be discouraged so far as preliminary observations go. Magnesium carbonate blocks purchased at different times may have reflection factors varying by at least several per cent. It would be an admirable thing for some scientific supply house to have in stock magnesium blocks of guaranteed texture and purity. Yet those who have the facilities of a photometric sphere need not rely on even such standards.

\section{A SIMPLER INSTRUMENT FOR COMMERCIAL USE}

The Nutting reflectometer requires neither lamp nor battery, a great advantage in a portable instrument. This advantage could possibly be obtained with other reflectometers by sacrificing accuracy for serviceability. It might, for example, be possible to accomplish this by the use of a translucent sphere or one in part transparent. In the reflectometer shown in Fig. 3 a mirror mounted so as to have sufficient freedom of rotation was substituted for the lamp to obviate the necessity of maintaining a light source. Unless, however, an intense external light source is available, the brightness is too low. With sunshine it works admirably. For practical uses, however, even simpler instruments might be used, for example, the one shown in Fig. 4. In this an opal glass sphere has a rather large opening covered with a flat disk. The disk has a circular aperture against which the test surface is placed surrounded by an annular area divided into sectors, having different known reflection factors. The test surface and sectors are equally illuminated by the sphere wall, and are viewed through an aperture, $W$. The brightness of the test surface is taken to be that of the sector whose brightness most nearly matches it. The use of 
a simple disk with graduated reflection factors was suggested mary years ago. It is not satisfactory where any great amount of specular reflection exists. In the present instrument such is not the case. Furthermore, a definite direction determined by angle $A$, Fig. 4, for observing is given. This direction might be chosen

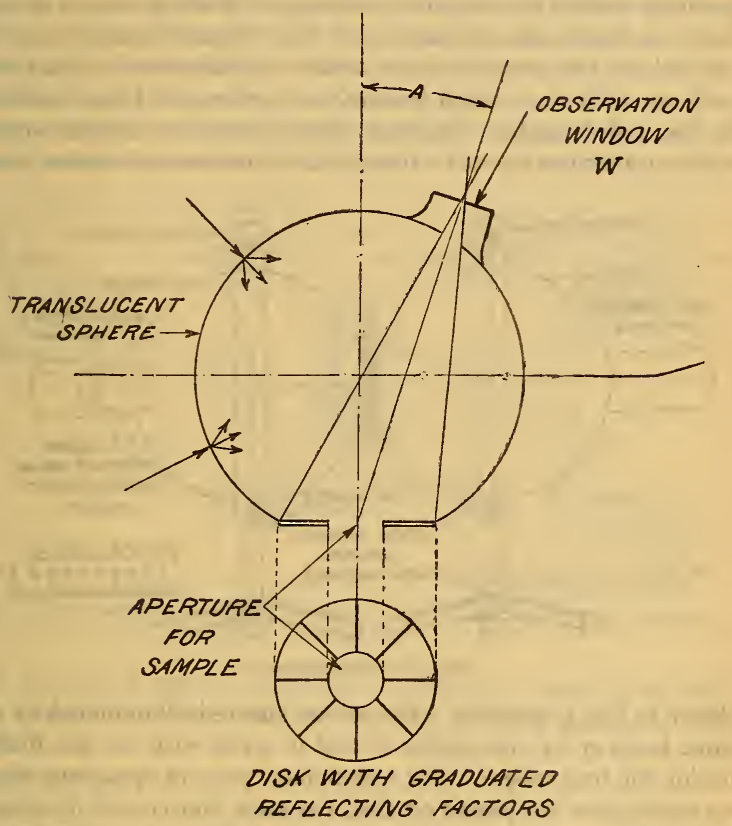

FIG. 4.-A simple reflectometer for commercial use

most favorably for many common materials met with in practice. Several workers ${ }^{33}$ seem to find that at some angle of emergence for normal illumination many surfaces have a reflection factor nearly equal to that of a perfectly diffusing surface of the same diffuse reflection factor.

2 A. H. Taylor finds this angle to be about $50^{\circ}$ for many surfaces. Prof. W. J. Drisko informs me that he has found the angle to be about $46^{\circ}$ for many surfaces. 


\section{A TRANSMISSOMETER}

The diffuse transmission factor is sometimes more important than the reflection factor, as in the case of photographic negatives and in diffusing glassware for illumination purposes. It is therefore desirable to have at hand a means of readily measuring this quantity with a fair degree of accuracy. While there are several ways in which the properties of the Ulbricht sphere may be utilized for this purpose, let it suffice to point out in detail here only one such way, which is merely an extension of that involved in the reflectometer described above. Another similar sphere with an aperture equal to that in the reflectometer sphere, as is

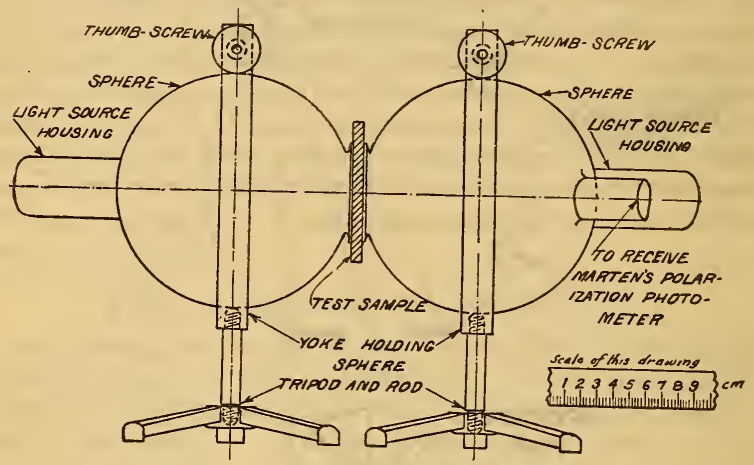

FIG. 5.-Transmissometer

shown in Fig. 5, is added. The second sphere is illuminated by the same lamp or by one similar to and in series with the one illuminating the first sphere. For a stationary piece of apparatus where no restrictions are placed on size, the same lamp could illuminate both spheres. The flux into the second sphere is controlled by means of a diaphragm with a variable aperture such as an iris, slit, or wedge. (See Fig. 6.) No exacting specifications are necessary. The aperture of the second sphere is screened from the direct flux, as in the reflectometer sphere. In this case, as in the reflectometer, we assume that the area of the aperture is so small that it will not affect the brightness of the sphere walls. This can probably always be sufficiently well attained. It is also assumed that the illuminated area is small. 
The use of this apparatus as a transmissometer is as follows: The transmission sample is placed over the aperture of the reflectometer as usual, and its reflection factor $\rho$ measured. The auxiliary sphere is then brought up so that the reverse side of the specimen covers its aperture. The brightness $b_{x}$ of the sample now is due to two factors, (I) the flux reflected from its front surface (which is illuminated by the walls of the first sphere), and (2) the flux transmitted by the specimen in consequence of the illumination by the walls of the second sphere. Let the walls of these spheres have a brightness of $b$ and $b_{1}$, respectively, and let the transmission factor of the sample be $\tau$. Then $b_{\mathrm{x}}=\rho b+\tau b_{1}$.

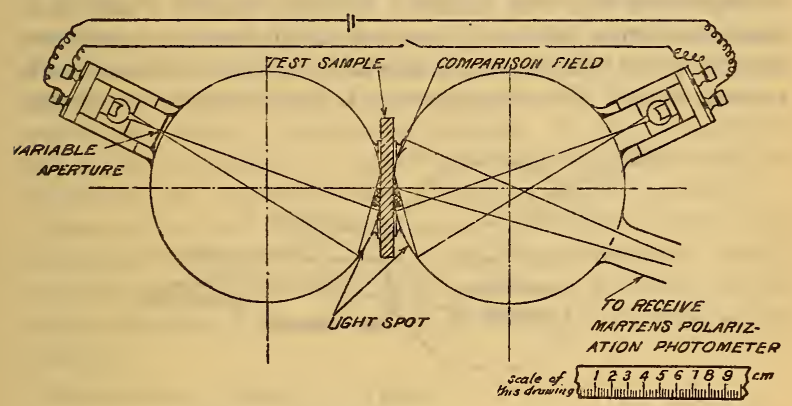

FIG. 6.-Transmissometer, showing position of light sources, test sample, photometer, and screens

The quantity $b_{1}$ may be measured in terms of $b$ once for all, and may have any value, but for the sake of simplicity, we shall make $b_{1}=b$.

Then $b_{x}=b(\rho+\tau)$.

$$
\frac{b_{x}}{b}=\rho+\tau \text {. }
$$

In this equation $\rho$ has been determined; $b_{x}$ is measured in terms of the brightness of the walls of the reflectometer sphere; $\tau$ is the factor desired; and $\frac{b_{\mathrm{x}}}{b}$ is measured and read off directly by means of the reflectometer. We see, therefore, that it is just as simple so far as manipulation is concerned to obtain the value of the combined reflection and transmission factors as it is to obtain the reflection factor alone. The difference of these two quantities gives the transmission factor. In this way one piece of laboratory 
apparatus enables us to obtain both the reflection factor and the transmission factor in an absolute manner, involving no calibration of a standard of transmission or reflection, no light standards, no measurement of distances, no manipulation of the specimen, and no electrical measuring instruments. One placing of the sample against the reflectometer is all.

Although the arrangement described appears to have certain advantages for laboratory purposes where duplication of costly photometric or spectrophotometric apparatus is not desired, or where it is desirable that such apparatus when once set up shall not be disturbed, it may be of interest to describe a few other arrangements that may be had. A simple method would be to insert the sample between the end of the photometer and sphere in such a way that one of the apertures only of the photometer is covered by it. The other aperture is illuminated by the sphere

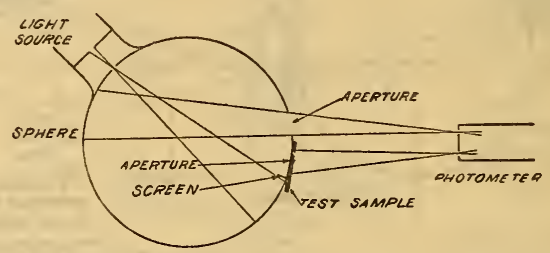

FIG. 7

wall, which in turn is illuminated by projecting a narrow beam into the sphere. Precautions must be taken to screen the test sample properly, as has been noted in the description of the reflectometer. Narrow limits are set to the size and shape of the sample that is to be tested.

A modification of this is shown in Fig. 7, where the photometer is removed from the sphere to a distance that allows greater freedom in the size and shape of the sample. In this case a comparison is made of the brightness $b$ of the sphere wall with the brightness $\tau b$ of the sample. The Martens polarization photometer measures $\tau$ directly.

In all these arrangements nothing novel can be claimed except in the use of the sphere rather than of an irregular inclosure or of superficial standards. The sphere affords two important essentials: (r) The kind of diffuse illumination desired in such measurements, and (2) the conditions for absolute determination. Neither of these can be so closely approximated by any other means. 
The combination of the sphere with the Martens photometer has been suggested and used by Goldberg ${ }^{34}$ in measuring the density of photographic plates. His suggestions differ from those given in this article regarding the general use of the sphere in measuring the transmission and reflection factors of substances in that in accordance with the latter the sphere is to be used not only to secure good diffuseness of the light but to obtain these factors in an absolute manner.

\section{USE OF REFLECTOMETER AND TRANSMISSOMETER WITH MONO- CHROMATIC LIGHT}

Although the instruments discussed above were primarily intended for mixed light, obviously they may also be used in determining the diffuse reflection and transmission factors of substances for spectral light. For this purpose the Koenig-Martens spectrophotometer is admirably adapted. In this connection, too, the use of the sphere, in ways described above, would make for definiteness and standardization, and afford absolute methods.

Most of this work was carried out with the cooperation of the searchlight investigation section of the Engineers, U. S. Army, who also made the executing of it possible by providing for it. Acknowledgment is also made to U. M. Smith for the illustrations of the text.

WASHINGTON, January 3r, I92I.

"Photographische korrespondenz; May-June, rgro. Densograph, ein registrierapparat zur messung der schwaerzung photographischer platten. 